\title{
An Experimental Study on Connectivity and Topology Control in Real Multi-hop Wireless Networks
}

\author{
Alvin Valera ${ }^{1}$ Pius W.Q. Lee ${ }^{1} \quad$ Yew Fai Wong ${ }^{2}$ Winston K.G. Seah ${ }^{1}$ \\ Hwee-Pink Tan ${ }^{1}$ Han Ju ${ }^{2}$ \\ ${ }^{1}$ Institute for Infocomm Research $\left(I^{2} \mathrm{R}\right)$, 1 Fusionopolis Way, \#21-01 Connexis, Singapore 138632 \\ ${ }^{2}$ National University of Singapore (NUS), 21 Lower Kent Ridge Rd, Singapore 119077 \\ \{acvalera,wqlee,winston,hptan\}@i2r.a-star.edu.sg \\ \{idmwyf,u0408764\}@nus.edu.sg
}

\begin{abstract}
Topology control by means of transmit power adjustment is a well-studied technique for improving the network capacity and energy efficiency of wireless ad hoc networks. In this paper, we investigate the feasibility of connectivity and topology control in a real IEEE $802.11 \mathrm{~b}$ testbed composed of inexpensive commercial-off-the-shelf (COTS) routers. Although topology control via transmit power adjustment is feasible based on analytical studies and emulations, it is difficult in practice for the range provided by COTS wireless routers.
\end{abstract}

\section{Categories and Subject Descriptors}

C.2.1 [Network Architecture and Design]: Wireless communication

\section{General Terms}

Measurement, Performance, Experimentation

\section{Keywords}

Topology control, Transmit power control, Wireless multihop routing

\section{INTRODUCTION}

An active area of research for many years in wireless sensor and ad-hoc networks is topology control by means of transmit power adjustment $[17,18,11]$. There are two main motivating factors for performing topology control: (i) to reduce radio interference in order to improve the capacity of the network; and (ii) to reduce energy consumption in order to extend the lifetime of energy-constrained networks.

Topology control by means of transmit power adjustment is based on the assumption that transmitting at higher power implies increasing effective communication range of nodes and vice versa. Accordingly, it is very commonly assumed

Permission to make digital or hard copies of all or part of this work for personal or classroom use is granted without fee provided that copies are not made or distributed for profit or commercial advantage and that copies bear this notice and the full citation on the first page. To copy otherwise, to republish, to post on servers or to redistribute to lists, requires prior specific permission and/or a fee.

WICON '08, November 17-19, 2008, Maui, Hawaii, USA

Copyright 2008 ICST 978-963-9799-36-3. (a)
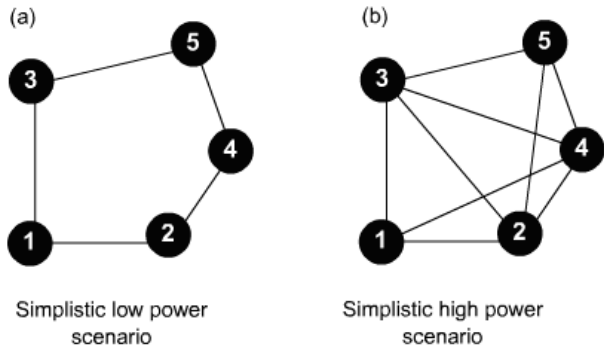

Figure 1: Simplistic views of the relationship between transmit power and network connectivity where circles represent nodes and lines represent bidirectional links. High transmit power causes the formation of more links.

that links between any two nodes either exist or they do not, as illustrated in Figure 1. Similarly, circular communication ranges with radii that vary with transmit powers are often assumed for convenience in analytical performance studies; this notion is also commonly adopted in simulation studies $[20,14,8]$

In recent years, experimental studies using IEEE 802.11 enabled devices such as notebooks and PDAs have become popular in the literature $[3,5,7,25]$. However, experimental studies on topology control and its impact on the performance of routing protocols remains largely lacking. Much of the recent work in topology control has focused on algorithms for determining and assigning the optimal transmit power that will enable the formation of a desired topology $[17,18,11]$. Less attention has been paid to answering an even more fundamental question: Can transmit power adjustment in real wireless devices indeed influence the network topology and bring about the benefits of topology control?

In this paper, we seek to address the above fundamental question and investigate the feasibility of controlling the network connectivity and topology by means of transmit power control on a real IEEE 802.11b testbed. We also examine its impact on the performance of both static routing and dynamic routing at the network layer.

The rest of the paper is organized in the following way. Section 2 discusses the experimental setup that is used in all our experiments. Section 3 presents our experimental findings. Section 4 discusses several related studies and Section 5 concludes the paper. 


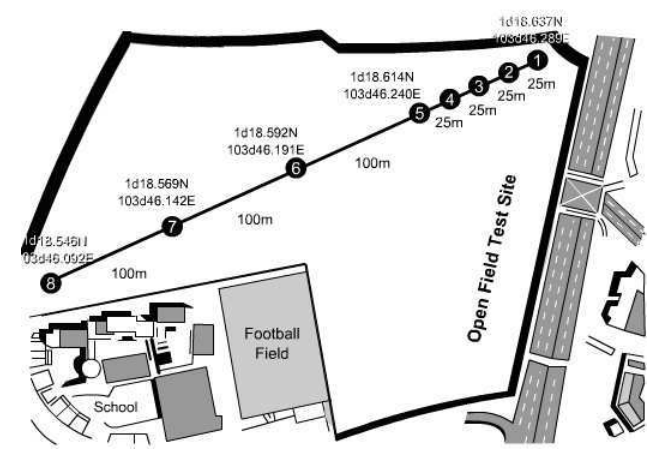

Figure 2: Open field test site deployment.

\section{EXPERIMENTAL SETUP}

We arrange a linear deployment of routers in an open field with inter-nodal distances of $25 \mathrm{~m}$ and $100 \mathrm{~m}$. This linear arrangement of nodes is typical in the monitoring of excavation sites in the construction of underground road systems, underground train networks and deep pipe installation works [19]. We also opted to study the string topology as it provides a scenario to easily analyze the network performance [22]. Part of our work is also commissioned by a Singapore systems engineering company to investigate wireless monitoring of an excavation site for the underground mass-rapid transit system. Current cabled monitoring solutions for underground monitoring pose challenging deployment issues including costs, space limitations in laying cables and interferences experienced by long cables [23]. In our wireless monitoring solution, wireless nodes (COTS routers) are at the ground level, where they are connected by short cables to strain gauge sensors attached to key load-bearing struts beneath ground level [19].

For clarity, wireless routers are labeled from $\mathrm{R} 1$ to $\mathrm{R} 8$ as shown in Figure 2 (with R1 at top-right corner) at the Test Site. Positioning is obtained using a combination of global positioning system (GPS) and measuring tape.

The Compex WP54G, 802.11b/g broadband routers are used as they are available commercially off-the-shelf (see Figure 3). The original Compex firmware is replaced with the open-source Linux-based OpenWRT distribution (Kami-

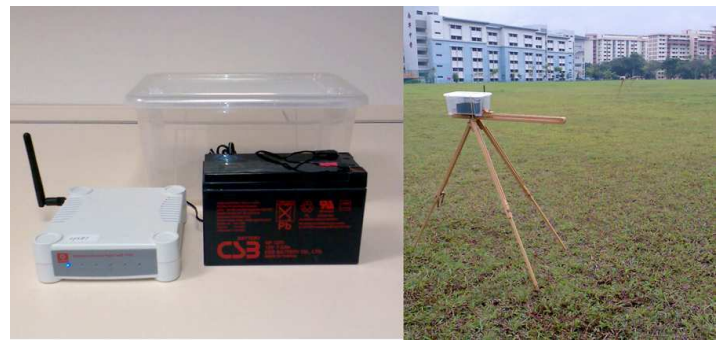

Figure 3: Wireless router and accessories used in the testbed. Left: One node is composed of one Compex WP54G router, one $12 \mathrm{~V}$ rechargeable lead battery and one plastic container to protect the hardware from rain. Right: Actual deployment of the node in the test site.
Table 1: Experimental Configurations

\begin{tabular}{|l|l|l|}
\hline 1 & Antenna height & $1 \mathrm{~m}$ from ground \\
\hline 2 & Antenna orientation & Vertically upright \\
\hline 3 & Power supply & $\begin{array}{l}\text { Rechargeable 12V Sealed } \\
\text { Lead Batteries }\end{array}$ \\
\hline 4 & Transmit power & $\begin{array}{l}1,4,7, \quad 10,13, \quad 16, \quad 19 \\
\mathrm{dBm}\end{array}$ \\
\hline 5 & Channel / Frequency & $6 / 2.437 \mathrm{GHz}$ \\
\hline 6 & MAC protocol & IEEE $802.11 \mathrm{~b}$ \\
\hline 7 & MAC RTS/CTS & Disabled \\
\hline 8 & MAC retry limit & 11 \\
\hline 9 & Unicast data rate & $11 \mathrm{Mb} / \mathrm{s}$ \\
\hline 10 & Sender application & UDP-based CBR \\
\hline 11 & Packet size & 40 and 1400 bytes \\
\hline 12 & Kamikaze version & 7.07 \\
\hline 13 & NTP version & $3.7 \mathrm{p} 1-1 ; 2003 \_194-2$ \\
\hline $14 \mathrm{a}$ & $\begin{array}{l}\text { AODV version } \\
\text { AODV gratuitous option } \\
\text { AODV HELLO interval }\end{array}$ & $\begin{array}{l}0.9 .3 \\
\text { Disabled } \\
\text { Yes, every 1s. }\end{array}$ \\
\hline $14 \mathrm{~b}$ & Static Routing Case & $\begin{array}{l}\text { Multihop via the path } \\
\text { R8 } \rightarrow \text { R7 } \rightarrow \text { R6 } \rightarrow \text { R5 } \\
\rightarrow \text { R1. }\end{array}$ \\
\hline
\end{tabular}

kaze 7.07) [4]. OpenWRT provides a fully writable filesystem with package management facilities to install other programs that are required by our project. It enables the installation of Uppsala University's AODV-UU, a popular implementation of the AODV [16] routing protocol. AODV is also one of the popular choices for wireless ad hoc routing in the literature. The network times across different routers are synchronized using the Network Time Protocol (NTP) [13] for the purpose of tracing packets. Most importantly, OpenWRT provides a tool called iwconfig that can be used to adjust the transmit power of the wireless interface card.

Table 1 summarizes our experimental configuration in greater detail. Antenna height is about $1 \mathrm{~m}$ from the ground, and orientation is vertically upright as shown in the inset picture of Figure 2. No other modifications to the antenna configuration are made. The IEEE 802.11b [21] is used as the medium access control (MAC) protocol. The channel is fixed at channel 6 which uses the $2.437 \mathrm{GHz}$ frequency. MAC RTS/CTS is disabled as it has been found to degrade network performance $[2,12]$. The MAC retry limit is 11 which is the default value in the device driver. For unicast transmissions, the data rate is fixed at $11 \mathrm{Mb} / \mathrm{s}$.

In all experiments involving traffic generation, a UDPbased constant bit rate (CBR) application is used to send data packets. Items $14 \mathrm{a}$ and $14 \mathrm{~b}$ are mutually exclusive. Only one of the routing schemes is active at any one time during our experiments.

\section{EXPERIMENTAL RESULTS}

In this section, we present the experimental results obtained from the IEEE 802.11b testbed described in Section 2. The data presented in this section were collected over several runs which spanned several days of experimentations. To determine the effect of transmit power on the network topology and routing performance, we measure the following performance metrics:

Packet Delivery Probability (PDP) This metric is de- 
PDP vs Sender-Receiver Distance of 40-byte UDP Packets

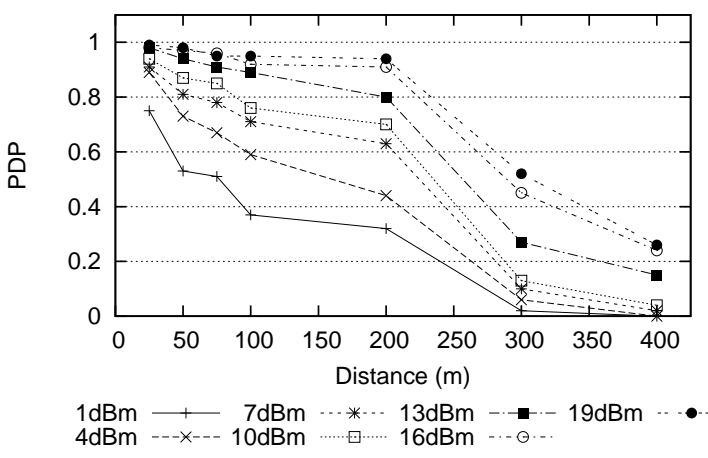

PDP vs Sender-Receiver Distance of 1400-byte UDP Packets

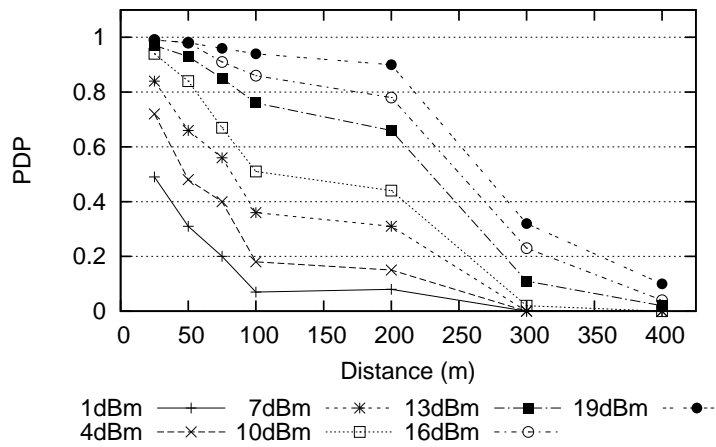

Figure 4: Packet delivery probability for different power levels over different distances from an emitting source node (R1): (a) using 40-byte UDP packets; and (b) using 1400-byte UDP packets.

fined as the fraction of all packets from a sender node that reach a receiver node at the data link layer.

Packet Delivery Ratio (PDR) This metric is defined to be the fraction of all data packets from a source node that reach a sink node at the application layer.

End-to-end Delay This metric is defined as the delay for every delivered data packet and is measured as the difference between the time the packet is received by the sink application and the time the packet is sent by the source application.

The PDP metric is used to ascertain the link quality while the PDR metric is used to ascertain the performance of network-layer routing. Logically, a high-PDP link is likely to provide better performance than a low-PDP link. A path composed of high-PDP links is likely to provide a better PDR than a path that is composed of low-PDP links.

We will frequently use the terms link and path in this paper. For clarity, we define a link as a direct wireless connection between two nodes. More specifically, we say that a link between nodes $A$ and $B$ exists if either one of the nodes can receive a fraction of the packets being sent by the other node. On the other hand, we define a path as a string of links from a source to a destination. A path can be composed of one or more links ${ }^{1}$.

\subsection{Transmit Power Adjustments to Control Network Topology}

\subsubsection{Interference-Free Experiment}

One major requirement of topology control is the ability to control the transmission range of the nodes in the network. This is indirectly achieved by adjusting the transmit power of the node's wireless transceiver. Lower transmit power implies shorter transmission range while higher transmit power implies longer transmission range.

\footnotetext{
${ }^{1}$ In the discussion, we will mention "short link" and "short path". A short link between $A$ and $B$ means that $A$ is physically "near" from $B$. A short path refers to a path with less number of links (or hops) but this does not necessarily mean that the links are short.
}

In static networks such as the one being studied in this paper, it is important to determine the critical transmission range (CTR) because dynamic adjustment of transmission range has been found to be infeasible using inexpensive wireless transceivers [18]. CTR is defined as the minimum transmission range that ensures high probability of network connectivity [18]. If the node positions are known in advance, then the CTR is simply the longest edge of the Euclidean minimum spanning tree [15]. For the string topology that is being studied in this paper (see Figure 2), the CTR is 100 $\mathrm{m}$.

In this experiment, our objective is to empirically determine the minimum transmit power needed to reach the CTR of $100 \mathrm{~m}$. We let node $\mathrm{R} 1$ transmit broadcast packets using a UDP-based CBR application at a rate of 4 packets/second. Two packet sizes are used, namely 40 bytes and 1400 bytes. The transmit power is varied using the values listed in Table 1. Each experiment at a given transmit power level runs for 10 minutes. All nodes R1, R2, R3, R4, R5, R6, R7 and $\mathrm{R} 8$ are switched on, with $\mathrm{R} 1$ as the only node sending out packets.

Figure 4 shows the packet delivery probability (PDP) at different sender-receiver distances as the transmit power is adjusted from $1 \mathrm{dBm}$ to $19 \mathrm{dBm}$ in $3 \mathrm{dBm}$ steps. In general, the PDP decreases as the sender-receiver distance increases for all power levels. Moreover, the PDP increases as the transmit power increases for all sender-receiver distance. In terms of the packet size, the 40-byte packets have higher PDP compared with the 1400-byte packets. These results are essentially in line with theoretical expectations.

In simpler terms, the results indicate that with transmit power of $1 \mathrm{dBm}$, transmit range can be up to $200 \mathrm{~m}$. At transmit power of $19 \mathrm{dBm}$, transmit range can be up to 400 $\mathrm{m}$, which effectively covers the entire network that we are studying in this paper.

Figure 4 shows several interesting features. Firstly, from $25 \mathrm{~m}$ to $100 \mathrm{~m}$, there is a considerable decrease in PDP especially for the lower transmit power values. Higher transmit power values seem to slow down the decrease in PDP within this range. Secondly, from $100 \mathrm{~m}$ to $200 \mathrm{~m}$, the PDP does not show any significant decrease. The decrease is less than $10 \%$ in all cases except for the 4 - $\mathrm{dBm}, 40$-byte packet case (see Figure 4(a)). We find this result surprising and would 
PDP vs Transmit Power of 100m Links (40-Byte Packets)

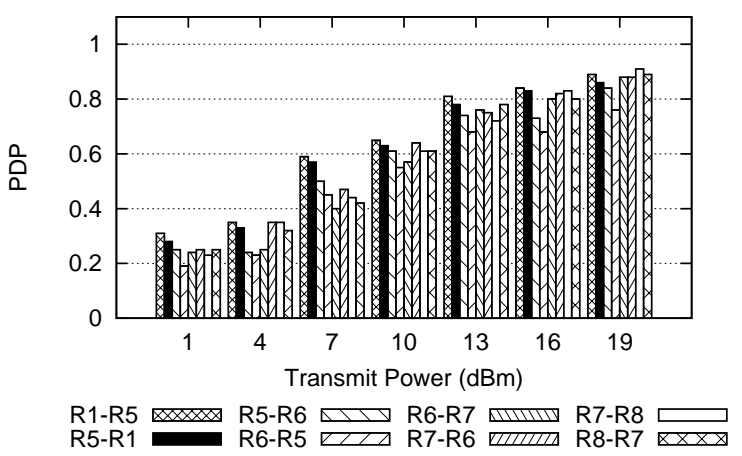

PDP vs Transmit Power of 100m Links (1400-Byte Packets)

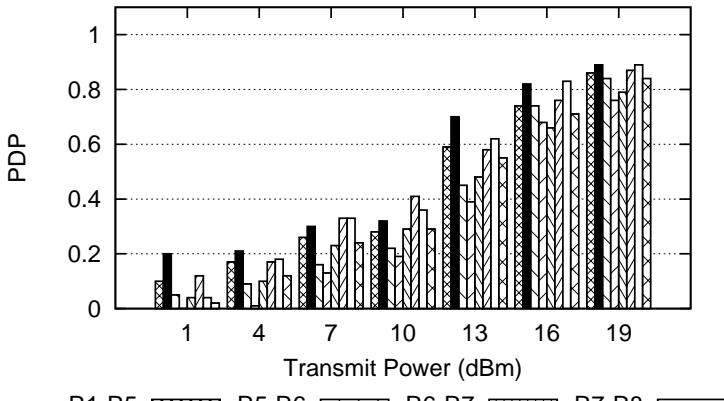

R1-R5 R5-R6 $₫ \backslash \mathrm{R}$ R-R7 MIII) R7-R8

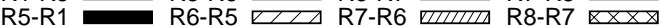
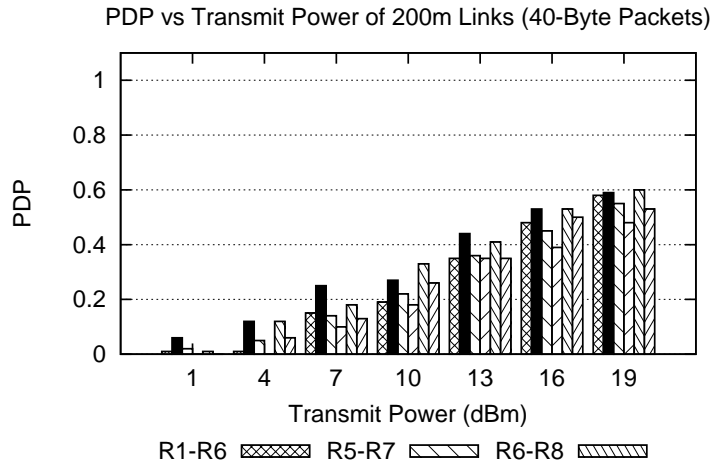

$\begin{array}{lll}R 1-R 6 & R 5-R 7 \\ \text { R6-R1 } & \text { R7-R5 R6-R8 }\end{array}$

PDP vs Transmit Power of 200m Links (1400-Byte Packets)

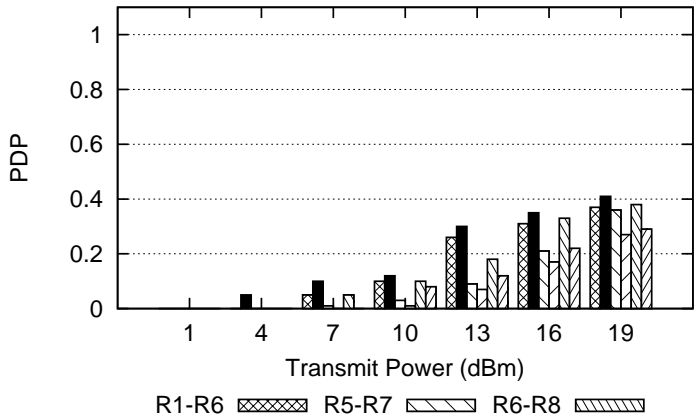
R6-R1 R7-R5 R8-R6 प्याप्य

Figure 5: Packet delivery probabilities (PDP) for different power levels: (a) 100m links, 40-byte UDP packets; (b) 200m links, 40-byte UDP packets; (c) 100m links, 1400-byte UDP packets; and (d) 200m links, 1400-byte UDP packets.

have expected the PDP at $200 \mathrm{~m}$ to be much lower than the PDP at $100 \mathrm{~m}$. Finally, from $200 \mathrm{~m}$ to $300 \mathrm{~m}$, the PDP plummets by at least $50 \%$ for the higher transmit power levels (13, 16 and $19 \mathrm{dBm})$.

From these results, it is clear that to achieve a sufficiently high PDP (greater than $70 \%$ ) at $100 \mathrm{~m}$, the transmit power required is at least $10 \mathrm{dBm}$ and $13 \mathrm{dBm}$ for 40-byte and 1400-byte packets, respectively. These power levels are also sufficient to achieve a high PDP at $200 \mathrm{~m}$. This finding is advantageous as it increases the degree of network connectivity at the same power level.

\subsubsection{Mutual Interference Experiment}

In the previous experiment, we determined the packet delivery probability at different sender-receiver distances. We also determined the minimum transmit power required to reach the critical transmitting range (CTR) of $100 \mathrm{~m}$. However, the results do not take into account the effect of interference or contention on PDP. In this experiment, we allow five nodes, R1, R5, R6, R7 and R8 (100 m apart) to transmit broadcast packets simultaneously. Nodes R2, R3, and R4 are switched off. The same transmit power settings, packet sizes, packet sending rate, and run time as in the previous experiment are used.

Figure 5 shows the packet delivery probability of all the links as the transmit power is adjusted from 1 to $19 \mathrm{dBm}$ in
$3 \mathrm{dBm}$ steps. Figures 5(a) and 5(c) show the PDP of links where the nodes are $100 \mathrm{~m}$ apart while Figures $5(\mathrm{~b})$ and $5(\mathrm{~d})$ show the PDP of links where the nodes are $200 \mathrm{~m}$ apart. (The plots for the 300 and $400 \mathrm{~m}$ links are not shown. For these cases, PDPs for these links are below 15\%.) Similar to the interference-free experiment, an increase in transmit power results in an increase in the PDP. For the $100 \mathrm{~m}$ links, the PDP for both 40-byte and 1400-byte packets reaches $90 \%$. However, note that the PDP of the bigger packets seems to increase at a slower pace compared with the PDP of the smaller packets as the transmit power is increased.

Interestingly, the links towards the nodes at the edge of the network $(\mathrm{R} 5 \rightarrow \mathrm{R} 1, \mathrm{R} 6 \rightarrow \mathrm{R} 1, \mathrm{R} 6 \rightarrow \mathrm{R} 8$, and $\mathrm{R} 7 \rightarrow$ R8) have the highest PDP compared with the other links on every transmit power level. This can be explained by the fact that the nodes R1 and R8 have fewer "neighbors" than the rest of the nodes (nodes $\mathrm{R} 1$ and $\mathrm{R} 8$ have no neighbor on one of their sides.) Hence, collisions are less likely to occur at these positions than in the "center" of the network.

Comparing this result with the results in the interferencefree experiment, one significant difference that can be observed is the lower PDP attained by the $200 \mathrm{~m}$ links. Note that in the interference-free experiment, the PDP peaks at $90 \%$ for the $200 \mathrm{~m}$ sender-receiver distance. However, in this experiment, the PDP for the $200 \mathrm{~m}$ links are all below $65 \%$, a decrease of at least $25 \%$. This significant decrease can be 


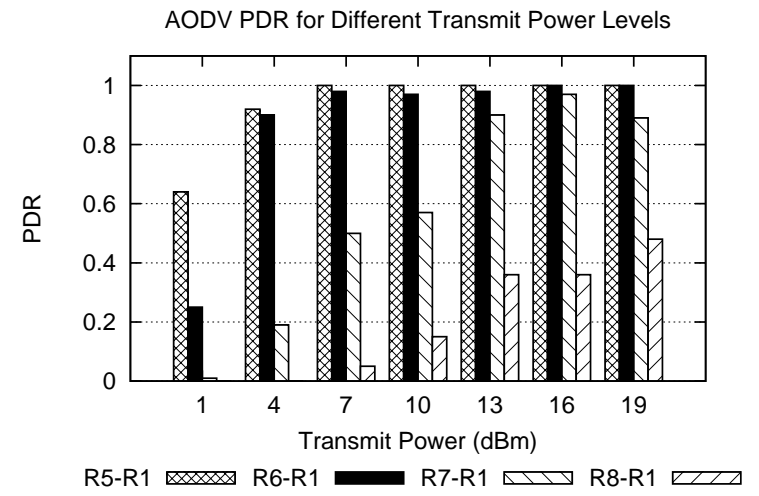

Static Routing PDR for Different Transmit Power Levels

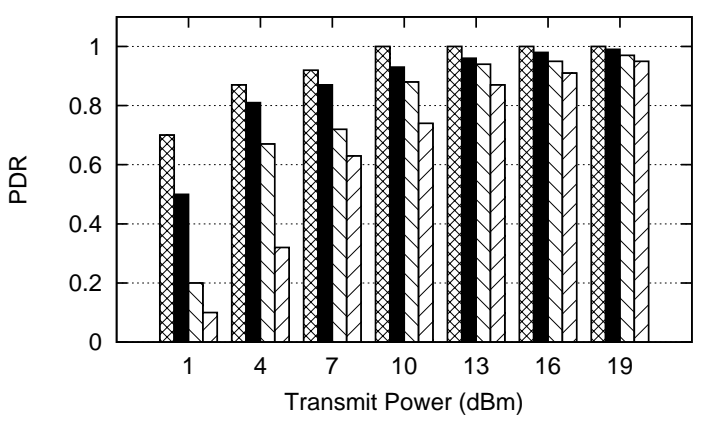

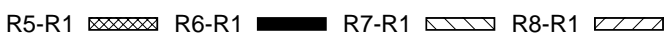

Figure 6: Packet delivery ratios (PDR) for (a) AODV and (b) Static Route over different power levels.

attributed to interference due to simultaneous transmissions by the five nodes. Note that the PDP of the $100 \mathrm{~m}$ links are also lower than in the interference-free case. However, the difference is not as significant as in the $200 \mathrm{~m}$ links. This result suggests that the effect of interference is worse for longer links. This can be due to the more prominent effect of the hidden node problem. Consider for example the 200 $\mathrm{m}$ link R8 $\rightarrow$ R6. From the position of node R8, node R 1 is highly likely to be hidden. Hence, node R 8 may simultaneously transmit a packet with R 1 causing a collision as far as R6 is concerned. Compare this with the $100 \mathrm{~m}$ link R7 $\rightarrow \mathrm{R} 6$. In this case, R7 is likely to sense transmissions from $\mathrm{R} 1$ thereby avoiding the packet collisions at R6.

\subsection{Impact on Network Layer Routing}

Up to this point, we have found out that transmit power adjustment indeed affects the packet delivery probability of the links. In this section, we examine whether these variations in PDP can provide an optimal point for the performance of network layer routing. Intuitively, it is possible to achieve optimum routing performance at around $10-13$ $\mathrm{dBm}$ since we determined that this is the required power to achieve a relatively high PDP for the CTR of $100 \mathrm{~m}$.

We focus our investigation on the performance of static routing and dynamic routing using AODV [16] when the transmit power is adjusted from $1 \mathrm{dBm}$ to $19 \mathrm{dBm}$. The experiment is configured such that nodes R5, R6, R7 and R8 (100 m apart) send 1400-byte UDP packets using a CBR application to node $\mathrm{R} 1$ at the rate of 32 packets per second. In contrast with the previous experiments, the data packets are sent as unicasts. Nodes R2, R3 and R4 are switched off. Each experiment at a given transmit power level runs for 10 minutes. The CBR source sends data packets only during the first 30 seconds of each minute. It does not send any packet on the remaining 30 seconds. This is done to force the routes discovered by AODV to timeout more frequently and hence ensure that AODV does not use the same path discovered at the beginning for the entire duration of the experiment.

In the preceding experiments involving simultaneous transmissions, the results indicate that choosing a shorter link is much better than a longer link, supporting previous findings in other experimental studies $[5,10,6,7]$. To verify this, we configured static routing to traverse the path via $R 8 \rightarrow R 7$ $\rightarrow \mathrm{R} 6 \rightarrow \mathrm{R} 5 \rightarrow \mathrm{R} 1$ (see item $14 \mathrm{~b}$ of Table 1 ).

\subsubsection{Packet Delivery Ratio}

Figures $6(\mathrm{a})$ and $6(\mathrm{~b})$ show the packet delivery ratio of 1400-byte CBR packets using AODV and static routing, respectively. The high PDR for the static route case supports our previous findings that using shorter links can provide higher packet delivery probability. Compared with AODV, static routing yielded higher PDR for all CBR flows and transmit power levels. The difference is more significant for the flows emanating from the nodes that are further away from the sink $(\mathrm{R} 7 \rightarrow \mathrm{R} 1$ and $\mathrm{R} 8 \rightarrow \mathrm{R} 1)$.

The low PDR of AODV especially from nodes $\mathrm{R} 7$ and $\mathrm{R} 8$ can be explained by Figure 7 which shows the the number of hops taken by successfully delivered packets.

Figures 7(a) and 7(b) show that all packets from R5 and R6 took one hop - that is, direct transmission to node R1 - for all the tested transmit power levels. The high PDR attained by the CBR flows from R5 and R6 is due to these nodes being $100 \mathrm{~m}$ and $200 \mathrm{~m}$ away, respectively, from the sink node R1. This finding is in line with our observation in the interference-free experiment that the PDP at $100 \mathrm{~m}$ and $200 \mathrm{~m}$ are almost equal for a given transmit power. We however need to reconcile this with the result from the mutual interference experiment where the PDP reaches only at most $40 \%$ for 1400 -byte packets for the $200 \mathrm{~m}$ links. There are two major reasons behind the high PDR from R6 to R1 in the AODV experiments: (i) the position of node R6 at the center of the network reduces the hidden node problem; and (ii) data packets are sent unicast in this experiment as opposed to broadcast in the mutual interference experiment.

We first elaborate on the first reason. Note that with respect to R6, all nodes in the network have low probability of being hidden. In addition, all the other nodes in the network also "sees" node R6 since they are at most $200 \mathrm{~m}$ away from R6. Hence, node R6 is least likely to send data packets that will experience collisions.

With regards to the second reason, in IEEE $802.11 \mathrm{~b}$, unicast packets are sent reliably, that is, with acknowledgments from the receiver and are re-transmitted when the sender fails to receive an acknowledgment from the receiver. Hence, the low PDP for the $200 \mathrm{~m}$ links observed in the mutual interference experiment does not necessarily mean that the 200 $m$ links are of marginal quality. The low PDP is mainly due 

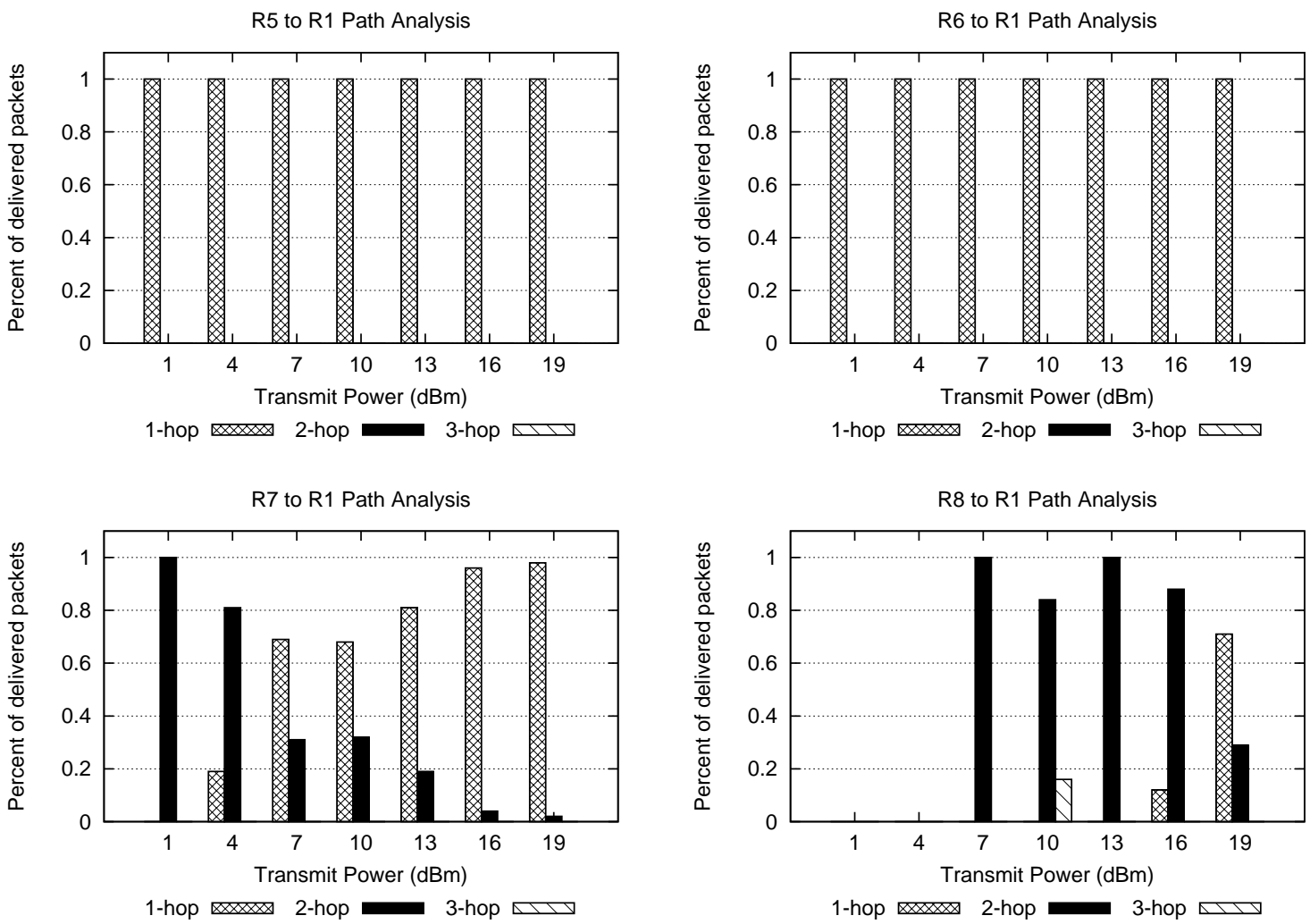

Figure 7: Number of hops taken by successfully delivered packets for different power levels: (a) R5 $\rightarrow$ R1; (b) R6 $\rightarrow \mathrm{R} 1$; (c) R7 $\rightarrow \mathrm{R} 1$; and (d) R8 $\rightarrow \mathrm{R} 1$.

to interference which can be countered to a certain extent by retransmissions at the MAC layer [5]. At the network layer, retransmissions result in increased delay.

Figures $7(\mathrm{c})$ and $7(\mathrm{~d})$ show the paths taken by the data packets from R7 and R8, respectively. From these figures, we can clearly see the effect of increasing transmit power on the paths chosen by AODV for routing data packets. For node R7, it initially uses two hops more than $70 \%$ of its packets at $1 \mathrm{dBm}$ and $4 \mathrm{dBm}$. Beyond $7 \mathrm{dBm}$, majority of R7's packets traverses a one hop or direct path to R1. Note that the link R7-R1 is $300 \mathrm{~m}$ long. From the results in the interference-free experiment (see Figure 4(b)), we can see that a $300 \mathrm{~m}$ link has a PDP of less than $40 \%$ for all transmit power levels. As such, using a one hop path from R7 to R1 causes significant packet loss especially at lower transmit power levels. Although MAC-layer retransmissions increase the PDR, the delay suffers as can be seen from the very high delay for the flow from R7 in Figure 8.

For R8, more than $80 \%$ of its packets traverse two hops until $16 \mathrm{dBm}$. At $19 \mathrm{dBm}$, more than $70 \%$ of its packets use one hop to R1, with the rest taking two hops. The low PDR of the flow from R8 is quite interesting since it used two hops most for most of the power levels. Expectedly, its $\mathrm{PDR}$ is higher if it relays its packets through R6 which is $200 \mathrm{~m}$ away. Based on the router table log, we determined that R8 uses R7 as the relay instead of R6 most of the time. As such, its PDR is also low since the link R7-R1 is $300 \mathrm{~m}$.
The explanations on why R8's PDR is very low and why its delay is very high is the same as in the preceding paragraph.

From the preceding discussions, we find one common cause of low PDR for the flows from R7 and R8: AODV used a $300 \mathrm{~m}$ link for relaying data packets when it would have been better to use a shorter link. Wireless ad hoc routing protocols such as OLSR [9] and AODV [16] use hello packets to determine its neighbors. These hello packets are periodically broadcasted by all nodes running the routing protocol. A node treats another node as a neighbor once it receives a hello packet. An AODV hello packet is small and is normally less than 40 bytes [16]. From the results in the interference-free experiment (see Figure 4(a)), we can see that a $300 \mathrm{~m}$ link has a non-zero PDP for all transmit power levels. Hence, no matter what transmit power value is chosen, AODV is likely to receive a hello packet from a node that is $300 \mathrm{~m}$ away.

The results shown in Figure 7 confirm that the paths chosen by AODV are quite different from the paths used in static routing. This accounts for the difference in the performance between AODV and static routing especially for the flows from R7 and R8. It is clear that the strategy used in static routing, which is to route packets over shorter links, provides better performance than that of AODV.

\subsubsection{Delay}

Figure 8 shows the average end-to-end delay for success- 

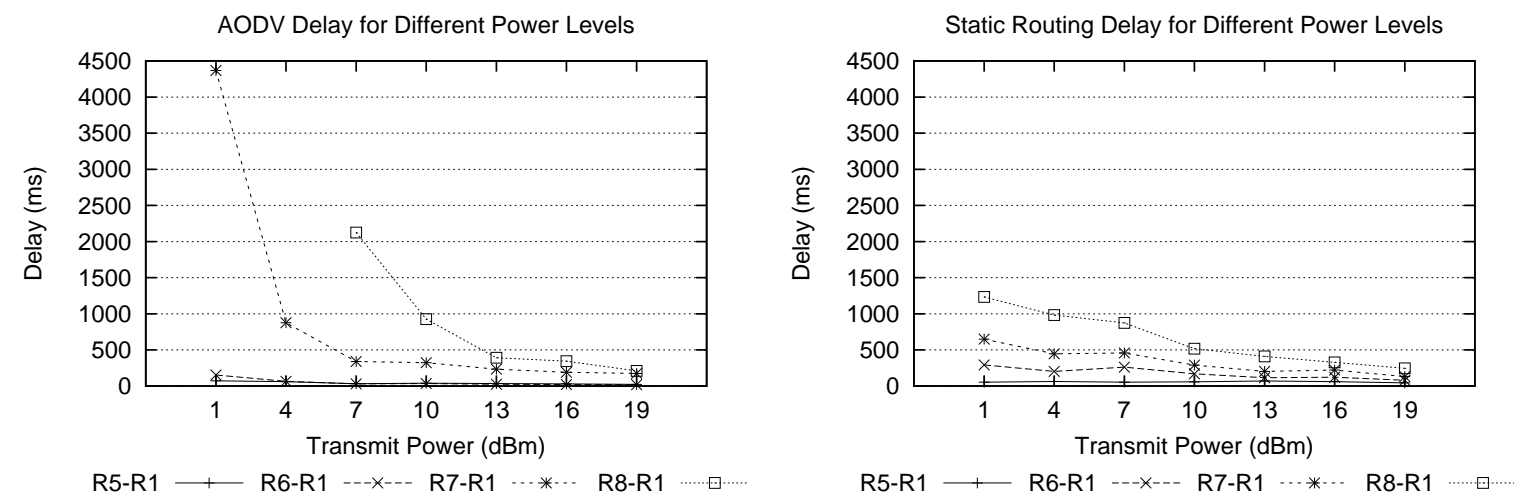

Figure 8: End-to-end delay for (a) AODV and (b) Static Route over different power levels. There are no delay values for 1 and $4 \mathrm{dBm}$ of the $\mathrm{R} 8 \rightarrow \mathrm{R} 1$ flow since no packets are successfully delivered in these cases.

fully delivered data packets. AODV seems to perform better than static routing for the CBR flows from R5 and R6. However, for the CBR flows from $\mathrm{R} 7$ and $\mathrm{R} 8$, static routing clearly has a much lower delay than that of AODV. In fact, the delay of AODV is extremely high at 4.4 seconds for the CBR flow from R7 at $1 \mathrm{dBm}$ and 2.1 seconds for the CBR flow from R8. As explained in the preceding discussion, the poor PDP of the links used for these flows resulted in MAC layer retransmissions which ultimately caused the delay to shoot up.

In the case of static routing, the delay consistently decreases as the transmit power increases. Expectedly, the delay is higher for flows that traverse longer paths since packets need to pass through more links. Each link traversal adds a certain amount of delay. This is a slight disadvantage of static routing.

To sum up, the packet delivery and delay results show that network layer routing performance, whether using dynamic routing or static routing, is indeed affected by transmit power adjustments. The optimum performance, however, can only be attained at the highest transmit power of $19 \mathrm{dBm}$. From the PDP results (see Figure 4), the network is already highly connected at this power level, that is, every node can "hear" every other node in the network. This result is not totally supportive of transmit power adjustment as a means to control the network topology for improving the performance of the network.

It can be argued that the benefits of topology control may become visible if we tested transmit powers higher than 19 $\mathrm{dBm}$. For example, it is reasonable to speculate that at $22 \mathrm{dBm}$, the PDRs may start to drop and the delays may start to rise. However, at such high powers, the network is most likely to be fully connected, with the $400 \mathrm{~m}$ link R8-R1 having a high PDP.

\section{RELATED WORK}

The subject of topology control is currently an active area of research in wireless multi-hop networks [17, 24, 18, 11]. However, existing work deals mostly with devising a topology control algorithm for optimizing the transmit power or range. Furthermore, these studies are mostly analytical or emulations at best. In this paper, we investigated the more basic question of whether topology control by means of transmit power adjustment is feasible in real wireless ad hoc networks.

There have been several measurement studies on the link characteristics and transmit power control capabilities of existing IEEE 802.11 devices [1, 2, 12]. Aguayo et al [1] studied the patterns and causes of packet loss in an IEEE 802.11b mesh network. Anastasi et al [2] studied the relationship of transmission rate with respect to throughput and carrier sensing range. Liese et al [12] studied several aspects of wireless mesh network design including multi-radio utilization, channel assignment and antenna placement. Our work is different from these studies as we focused our experiments on determining whether or not transmit power adjustments can influence the topology formation.

\section{CONCLUSIONS AND FUTURE WORK}

Based on our experimental studies conducted on a real IEEE 802.11b-based wireless ad hoc network, we make the following conclusions:

- Adjusting the transmit power affects the packet delivery probability at difference source-receiver distances. Lower transmit power results in lower delivery probability while higher transmit power yields higher delivery probability.

- Choosing a shorter wireless link results in better delivery probability. Longer links are more prone to packet collisions due to a more prominent effect of the hidden node problem.

- Although transmit power adjustment can influence topology formation, its impact on the performance of AODV is limited. The main problem is that transmit power adjustment does not eliminate long unreliable links.

In the IEEE $802.11 \mathrm{~b}$ wireless ad hoc network testbed that we used for our experiments, the optimum performance of both static and dynamic routing can only be attained at the highest transmit power of $19 \mathrm{dBm}$ or $84 \mathrm{~mW}$. At this power level, the network is already highly connected with all nodes in transmit range of one another. This finding suggests that 
topology control via transmit power adjustment is not effective in improving the performance of the network since we ultimately have to use the highest power to achieve the optimum performance. Obviously, transmitting at high power is incompatible with one of the goals of topology control which is energy efficiency.

There are several issues that need to be examined in connection with topology control via transmit power adjustment. One aspect that we are currently investigating is the performance of the network at transmit powers beyond 19 $\mathrm{dBm}$. Another important issue that we are studying is the effect of transmit power adjustment on routing metrics that use packet delivery probability as a link quality estimate. The performance of these metrics, when used in conjunction with topology control, is an interesting subject that can be further explored. Another aspect that can be investigated is the performance when RTS/CTS is enabled. Note that in this study, we disabled RTS/CTS since it degrades network performance $[2,12]$. However, we observed several cases where the hidden node problem occurred.

\section{REFERENCES}

[1] D. Aguayo, J. Bicket, S. Biswas, G. Judd, and R. Morris. Link-level measurements from an $802.11 \mathrm{~b}$ mesh network. In Proc. ACM SIGCOMM 2004, Portland, Oregon, Aug. 2004.

[2] G. Anastasi, E. Borgia, M. Conti, and E. Gregori. Wi-fi in ad hoc mode: a measurement study. Proc. IEEE PerCom 2004, pages 145-154, Mar. 2004.

[3] E. Borgia and F. Delmastro. Effects of unstable links on AODV performance in real testbeds. EURASIP Journal on Wireless Communications and Networking, 2007(1), Jan. 2007.

[4] OpenWRT Community. Openwrt wireless freedom. http://openwrt.org/.

[5] D. De Couto, D. Aguayo, J. Bicket, and R. Morris. A high-throughput path metric for multi-hop wireless routing. In Proc. ACM MOBICOM 2003, pages 134-146, San Diego, California, Sept. 2003.

[6] D. De Couto, D. Aguayo, B. Chambers, and R. Morris. Performance of multihop wireless networks: shortest path is not enough. SIGCOMM Comput. Commun. Rev., 33(1):83-88, Jan. 2003.

[7] R. Draves, J. Padhye, and B. Zill. Comparison of routing metrics for static multi-hop wireless networks. SIGCOMM Comput. Commun. Rev., 34(4):133-144, Oct. 2004

[8] C. Gui and P. Mohapatra. Power conservation and quality of surveillance in target tracking sensor networks. In Proc. ACM MOBICOM 2004, pages 129-143, Philadelphia, PA, USA, Sept. 2004.

[9] P. Jacquet, P. Muhlethaler, T. Clausen, A. Laouiti, A. A. Qayyum, and L. Viennot. Optimized link state routing protocol for ad hoc networks. In Proc. IEEE INMIC 2001, pages 62-68, Dec. 2001.

[10] V. Kawadia and P. Kumar. A cautionary perspective on cross-layer design. IEEE Wireless Communications Magazine, 12(1):3-11, Feb. 2005.

[11] F. Khadar and D. Simplot-Ryl. Connectivity and topology control in wireless ad hoc networks with realistic physical layer. In Proc. ICWMC 'O'\%, page 49, Washington, DC, USA, Mar. 2007. IEEE Computer
Society.

[12] S. Liese, D. Wu, and P. Mohapatra. Experimental characterization of an $802.11 \mathrm{~b}$ wireless mesh network. In Proc. ACM IWCMC 2006, pages 587-592, New York, NY, USA, 2006. ACM.

[13] D. Mills. Network time protocol version 4 protocol and algorithms specification, May 2007. IETF RFC 1305.

[14] R. Montemanni and L. M. Gambardella. Power-aware distributed protocol for a connectivity problem in wireless sensor networks. In Self-Star Properties in Complex Information Systems, Lecture Notes in Computer Science (LNCS), volume 3460, pages 403-416. Springer, May 2005.

[15] M. Penrose. The longest edge of the random minimal spanning tree. The Annals of Applied Probability, 7(2):340-361, May 1997.

[16] C. Perkins, E. Belding-Royer, and S. Das. Ad hoc on-demand distance vector (AODV) routing, Oct. 2003. IETF RFC 3561.

[17] R. Ramanathan and R. Rosales-Hain. Topology control of multihop wireless networks using transmit power adjustment. In Proc. IEEE INFOCOM 2000, volume 2, pages 404-413, mar 2000.

[18] P. Santi. Topology control in wireless ad hoc and sensor networks. ACM Comput. Surv., 37(2):164-194, June 2005.

[19] W.K.G. Seah and G.H. Tan. Wireless multihop networks in mission critical realtime monitoring and alerts for construction sites. In Proc. 3rd Intl. Conf. on Structural Health Monitoring of Intelligent Infrastructure (SHMII-3), Vancouver, Canada, Nov. 2007.

[20] S. Slijepcevic and M. Potkonjak. Power efficient organization of wireless sensor networks. In Proc. IEEE ICC 2001, volume 2, pages 472-476, Helsinki, Finland, June 2001.

[21] IEEE Computer Society. ANSI/IEEE std 802.11-1999 (part 11: Wireless LAN medium access control (MAC) and physical layer (PHY) specifications), June 1999.

[22] M. Takai, R. Bagrodia, K. Tang, and M. Gerla. Efficient wireless network simulations with detailed propagation models. Wireless Networks, 7(3):297-305, May 2001.

[23] G.H. Tan and T. Tan. Causes of false alerts in real time monitoring and alert systems in deep excavation sites. In Proc. 4th Underground Singapore 2007 Conference, Singapore, Nov. 2007.

[24] R. Wattenhofer, L. Li, P. Bahl, and Y.-M. Wang. Distributed topology control for power efficient operation in multihop wireless ad hoc networks. In Proc. IEEE INFOCOM 2001, volume 3, pages 1388-1397, apr 2001.

[25] N. Xu, S. Rangwala, K. K. Chintalapudi, D. Ganesan, A. Broad, R. Govidan, and D. Estrin. A wireless sensor network for structural monitoring. In Proc. ACM SENSYS 2004, pages 13-24, Baltimore, MD, USA, Nov. 2004. 